\section{(2) OPEN ACCESS}

- Additional material is published online only. To view please visit the journal online (http://dx.doi.org/10.1136/ thoraxjnl-2018-211589).

${ }^{1}$ Cicely Saunders Institute of Palliative Care, Policy and Rehabilitation, King's College London, London, UK

${ }^{2}$ School of Health Sciences, University of East Anglia, Norwich, UK

${ }^{3}$ Department of Oncology, University of Cambridge,

Cambridge, UK

${ }^{4}$ NIHR Respiratory Biomedical Research Unit, Royal Brompton and Harefield NHS Foundation Trust and Imperial College, Harefield, UK

${ }^{5}$ Harefield Pulmonary Rehabilitation Unit, Harefield Hospital, Harefield, UK

\section{Correspondence to}

Dr Matthew Maddocks, Cicely Saunders Institute of Palliative Care, Policy and Rehabilitation, King's College London, London SE5 9PJ, UK;

matthew.maddocks@kcl.ac.uk

LJB and SM are joint first authors.

Received 26 January 2018 Revised 9 October 2018 Accepted 22 October 2018

Check for updates

(C) Author(s) (or their employer(s)) 2019. Re-use permitted under CC BY. Published by BMJ.

To cite: Brighton $L$, Miller $\mathrm{S}$, Farquhar $\mathrm{M}$, et al. Thorax 2019;74:270-281.

\title{
Holistic services for people with advanced disease and chronic breathlessness: a systematic review and meta-analysis
}

\author{
Lisa Jane Brighton, ${ }^{1}$ Sophie Miller, ${ }^{1}$ Morag Farquhar, ${ }^{2}$ Sara Booth, ${ }^{3}$ Deokhee $\mathrm{Yi}^{1}{ }^{1}$ \\ Wei Gao, ${ }^{1}$ Sabrina Bajwah, ${ }^{1}$ William D-C Man, ${ }^{4,5}$ Irene J Higginson, ${ }^{1}$ \\ Matthew Maddocks ${ }^{1}$
}

\begin{abstract}
Background Breathlessness is a common, distressing symptom in people with advanced disease and a marker of deterioration. Holistic services that draw on integrated palliative care have been developed for this group. This systematic review aimed to examine the outcomes, experiences and therapeutic components of these services.

Methods Systematic review searching nine databases to June 2017 for experimental, qualitative and observational studies. Eligibility and quality were independently assessed by two authors. Data on service models, health and cost outcomes were synthesised, using meta-analyses as indicated. Data on recipient experiences were synthesised thematically and integrated at the level of interpretation and reporting.

Results From 3239 records identified, 37 articles were included representing 18 different services. Most services enrolled people with thoracic cancer, involved palliative care staff and comprised 4-6 contacts over 4-6 weeks. Commonly used interventions included breathing techniques, psychological support and relaxation techniques. Meta-analyses demonstrated reductions in Numeric Rating Scale distress due to

\section{Key messages}

What is the key question?

- What are the outcomes, recipients' experiences and therapeutic components of holistic services for chronic breathlessness in people with advanced disease?

What is the bottom line?

- Overall these services reduce patient distress due to breathlessness and may improve psychological outcomes of anxiety and depression.

- Despite wide variability in content and delivery, recipients value tailored interventions and expert staff providing person-centred, dignified care.

Why read on?

- This is the first review to synthesise available quantitative and qualitative evidence around holistic services triggered by breathlessness, which may serve as an appropriate referral indicator for early integration of palliative care.
\end{abstract} breathlessness ( $n=324$; mean difference (MD) -2.30 , $95 \% \mathrm{Cl}-4.43$ to $-0.16, \mathrm{p}=0.03$ ) and Hospital Anxiety and Depression Scale (HADS) depression scores $(n=408$, $\mathrm{MD}-1.67,95 \% \mathrm{Cl}-2.52$ to $-0.81, \mathrm{p}<0.001$ ) favouring the intervention. Statistically non-significant effects were observed for Chronic Respiratory Questionnaire (CRQ) mastery $(n=259, \mathrm{MD} 0.23,95 \% \mathrm{Cl}-0.10$ to $0.55, p=0.17)$ and HADS anxiety scores $(n=552, M D$ $-1.59,95 \% \mathrm{Cl}-3.22$ to $0.05, \mathrm{p}=0.06$ ). Patients and carers valued tailored education, self-management interventions and expert staff providing person-centred, dignified care. However, there was no observable effect on health status or quality of life, and mixed evidence around physical function.

Conclusion Holistic services for chronic breathlessness can reduce distress in patients with advanced disease and may improve psychological outcomes of anxiety and depression. Therapeutic components of these services should be shared and integrated into clinical practice. Registration number CRD42017057508.

\section{INTRODUCTION}

Breathlessness is a common and distressing symptom of chronic disease, affecting almost all people with chronic respiratory disease, ${ }^{1}$ the majority with heart disease or cancer, ${ }^{1}$ and significant proportions of those with renal disease, neurological conditions or HIV/AIDS. ${ }^{12}$ With our ageing population and increasing multimorbidity, ${ }^{3}$ the number of people affected by breathlessness worldwide will rise. Breathlessness increases as disease progresses ${ }^{4}$ and often becomes chronic (ie, it persists despite optimal treatment of the underlying disease ${ }^{5}$ ). The symptom can result in fear, sleep disturbance, social Breathlessness also occurs alongside other troublesome symptoms such as cough, fatigue and anxiety, and serves as a marker of overall symptom burden and deterioration. ${ }^{89}$

There are limited pharmacological treatments for breathlessness: moderate evidence supports use of low-dose opioids, ${ }^{10} 11$ and there is little to support use of benzodiazepines. ${ }^{12}$ As these approaches do not address the psychosocial problems that underlie and perpetuate the symptom, ${ }^{13}$ non-pharmacological treatments take priority. In many people, breathlessness is successfully managed via isolation and disability for patients and carers. ${ }^{67}$ 
rehabilitation services that incorporate exercise training, education and behaviour change interventions. ${ }^{14} 15$ Pulmonary rehabilitation, for example, improves functional status and quality of life, and is a cornerstone of best standard breathlessness care. ${ }^{14}$ However, issues with referral, uptake and completion limit reach, particularly to people with the most advanced disease with high levels of disability. 61617

Holistic services are emerging, designed specifically for those with advanced disease and chronic breathlessness. ${ }^{18-21}$ These typically draw on palliative care, but with integrated working from multiple specialties and professional groups. Treatments are selected based on the physical, psychological, social and spiritual needs of individual patients, and their families or carers. Individual studies suggest a positive impact on health outcomes. ${ }^{18-21}$ For example, an integrated palliative and respiratory care service improved breathlessness mastery, and suggested a potential survival advantage. ${ }^{21}$ Recent international guidelines subsequently advocate for early integration of palliative care in people experiencing chronic disease, ${ }^{22} 23$ and refractory and/or distressing breathlessness may serve as an appropriate referral indicator, especially in non-cancer conditions where prognostication causes delays. ${ }^{24}$ However, the evidence base to guide practice and policy is poorly understood.

We therefore aimed to synthesise available evidence around holistic breathlessness services for people with advanced disease. Our objectives were to describe structures and therapeutic components; determine clinical and cost-effectiveness; and understand patients' and carers' experiences of these services.

\section{METHODS}

\section{Design and registration}

This systematic review and meta-analysis was conducted and reported in accordance with the Preferred Reporting Items for Systematic Reviews and Meta-Analyses (PRISMA) statement ${ }^{25}$; the protocol was prospectively registered (PROSPERO: CRD42017057508). ${ }^{26}$

\section{Inclusion and exclusion criteria \\ Participants}

Adults experiencing breathlessness related to advanced disease, including cancer (advanced local or metastatic), chronic respiratory disease (GOLD stage III-IV/grade C-D), heart failure (New York Heart Association stage III-IV) or progressive neurological conditions. Studies were eligible if $\geq 50 \%$ of participants met these definitions.

\section{Interventions and comparators}

In the absence of a standard definition, we defined holistic breathlessness services as those where patients are enrolled due to their breathlessness (not their diagnosis); drawing on skills from multiple specialties and disciplines; using a holistic approach encompassing non-pharmacological and pharmacological interventions as indicated; and supporting self-management. Interventions were excluded if they did not specifically target patients with breathlessness; or used single treatments (eg, breathing training alone). Pulmonary rehabilitation and disease-specific services (eg, integrated respiratory care) were deemed outside the scope of this review. Exclusively targeted service provider or carer interventions were excluded. All comparators were considered.

\section{Outcomes}

Health outcomes included breathlessness intensity, affect and impact domains ${ }^{27}$; anxiety and depression; physical function; health status or quality of life; and survival. Cost outcomes of

\section{Box 1 Search strategy}

\section{Electronic searches}

The following electronic databases were searched from their inception up to 2 June 2017:

- British Nursing Index

- CINAHL

- Cochrane Database of Systematic Reviews

- Central Register of Controlled Trials

- Database of Abstracts of Reviews of Effectiveness

- EMBASE

- MEDLINE

- PsycINFO

- Science Citation Index Expanded

Search terms were informed by literature scoping and information specialists, and piloted to ensure inclusivity. Subject headings and free text terms were combined to search for population and intervention terms (online supplementary appendix 1 shows the MEDLINE strategy).

\section{Handsearching}

Reference lists of retrieved studies and relevant reviews, citations, textbooks and voluntary sector materials were searched, and we contacted active researchers for unpublished data/grey literature. No language or publication status restrictions were imposed.

\section{Screening}

Records were imported into Endnote $X 7^{56}$ and duplicates removed. Two authors (SM, LB/MM) screened titles and abstracts for relevance, and independently assessed full texts of potentially eligible studies against eligibility criteria. Disagreements were resolved by discussion, and consultation with a third author $(\mathrm{IH})$ to reach consensus.

interest included service costs and utilisation, and quality-adjusted life-years (QALYs) derived from generic quality of life measures (eg, EuroQol-5D). Experience outcomes included patient and carer perspectives.

\section{Designs}

Randomised controlled trials (RCTs) and non-RCTs, observational studies and qualitative studies were included. Narrative reviews, opinion papers and case series with $<5$ participants were excluded. Our search strategy is shown in Box 1.

\section{Quality assessment}

Two authors (LB, MM) independently assessed the quality of included studies using the Standard Quality Assessment Criteria for Evaluating Primary Research Papers (QualSyst), ${ }^{28}$ which contains checklists for quantitative and qualitative studies. For mixedmethod studies, both checklists were used. QualSyst scores are summarised as a percentage score of applicable items. Information to aid quality assessment was obtained from primary, secondary and protocol articles. For RCTs we also assessed risk of bias using the Cochrane Collaboration tool. ${ }^{29}$

\section{Data extraction and analysis}

Data were extracted by one author $(\mathrm{SM} / \mathrm{LB})$ using a predesigned electronic form and checked by a second author to ensure rigour (LB/MM). Data were extracted on service characteristics (staff; contacts; duration; interventions; target population), study information (country; authors; year; design) and outcomes. Where additional information was needed for inclusion in 
meta-analysis, authors were contacted. For experience data, all text (including quotations) under the headings of 'results' or 'findings' in qualitative or mixed-methods studies were imported verbatim into qualitative data software (NVIVO V.12). ${ }^{30}$

Service characteristics and details of associated studies were tabulated. Component interventions were tabulated and summarised narratively. Data from controlled studies were included to estimate effectiveness. Outcomes were analysed as continuous data where possible. Mean differences (MDs) between intervention and comparator groups were reported with 95\% CIs. Where data permitted, meta-analysis was performed using random-effects models, and heterogeneity assessed using the $\mathrm{I}^{22}$ statistic. In all cases, individual studies were only represented once within each analysis. Sensitivity analyses excluded studies with high risk of bias $(<70 \%$ QualSyst score) and removed outliers where substantial heterogeneity $\left(\mathrm{I}^{2}>75 \%\right)^{31}$ was present. We planned funnel plots to assess reporting bias if $\geq 10$ studies were included. ${ }^{32}$ Additional findings were summarised narratively.

Qualitative data were coded line by line, and descriptive themes were developed encompassing the themes or codes of primary studies. From these, new analytical themes going beyond presentation of primary data were generated. ${ }^{33}$ Particular attention was paid to similarities and differences across studies, and divergent cases. Multiple stakeholders (researchers, patient/carer representatives, clinicians involved in delivery of services) reviewed the analysis and interpretation to ensure comprehensiveness and increase validity. Data were integrated at the level of interpretation and reporting.

\section{RESULTS}

Of 3239 unique records identified and 56 full texts screened, 37 articles were eligible for inclusion (figure 1). Articles were published in the period 1996-2017 (27 since 2010) and related to 18 separate holistic breathlessness services: 12 based in the UK, 3 in Canada and 1 each in Australia, Germany and Hong Kong.

\section{Service characteristics}

Thirty-three articles were included in the descriptive synthesis (tables 1 and 2). Most of the services (12 of 18) were delivered to people with advanced cancer and used a mixture of face-to-face and phone contacts (median 4-6, range 1-12) and were short term, usually over 4-6 weeks (range 1-12; table 1). Service providers included doctors, nurses, physiotherapists and

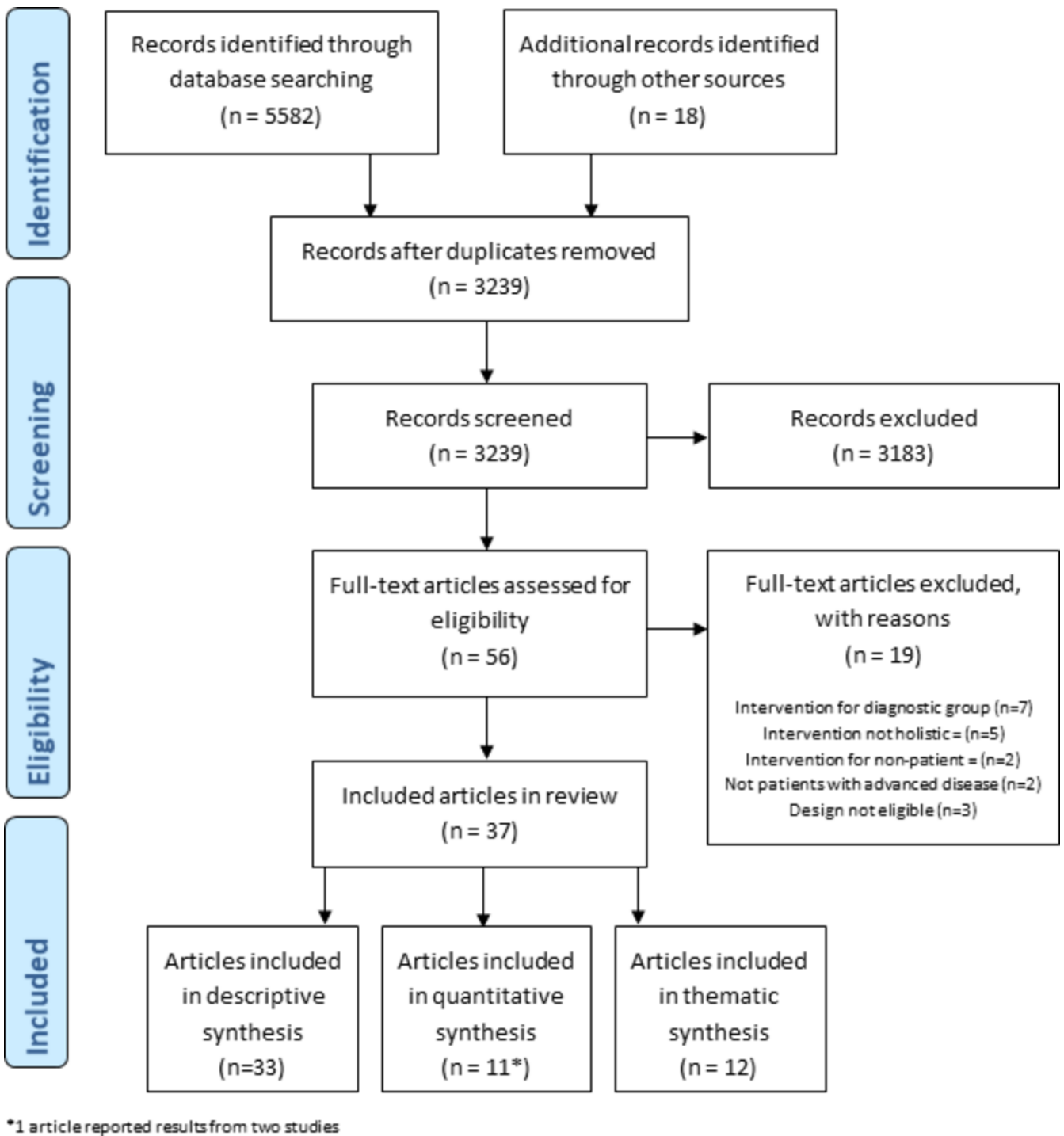

Figure 1 Preferred Reporting Items for Systematic Reviews and Meta-Analyses flow diagram. 


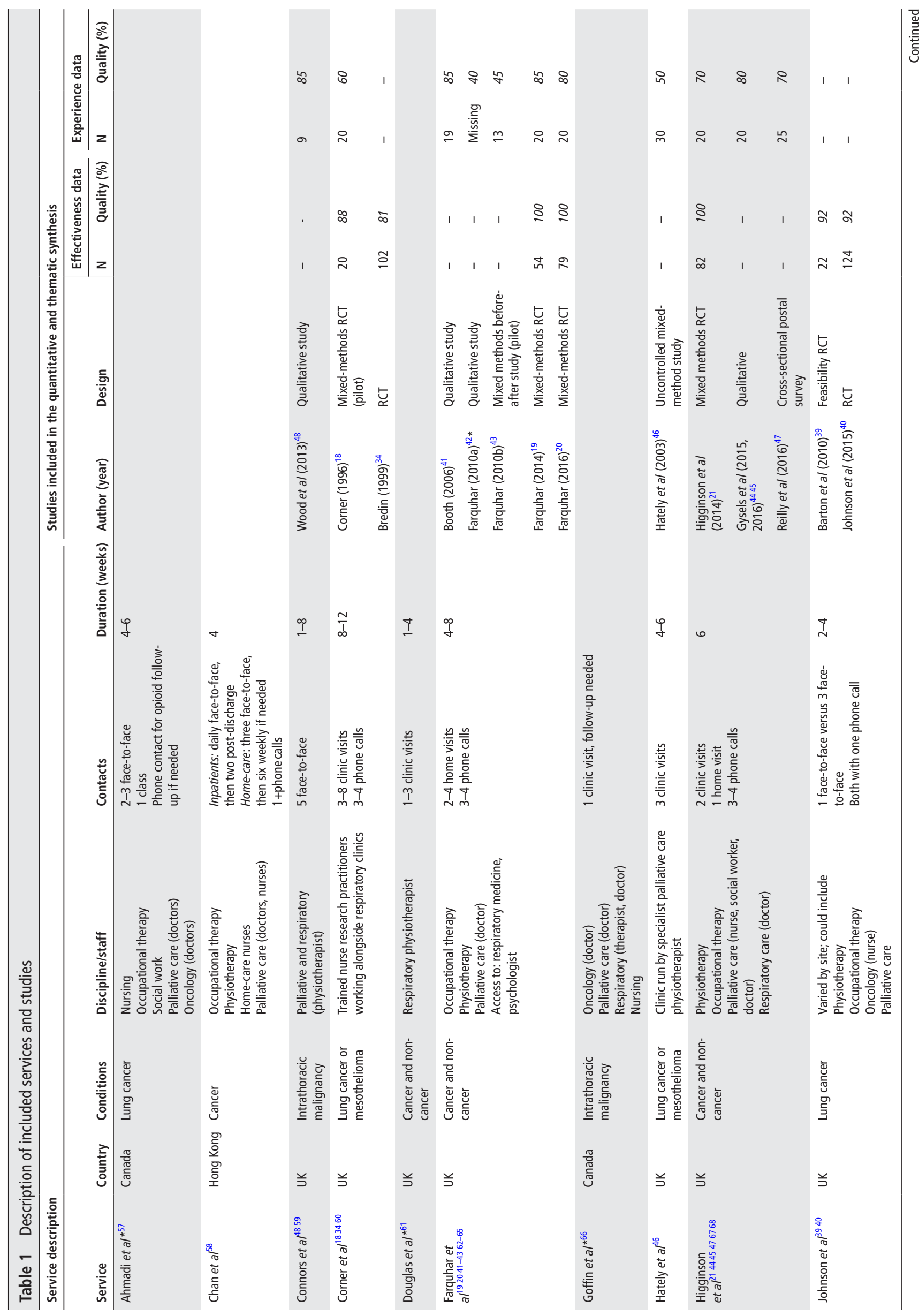




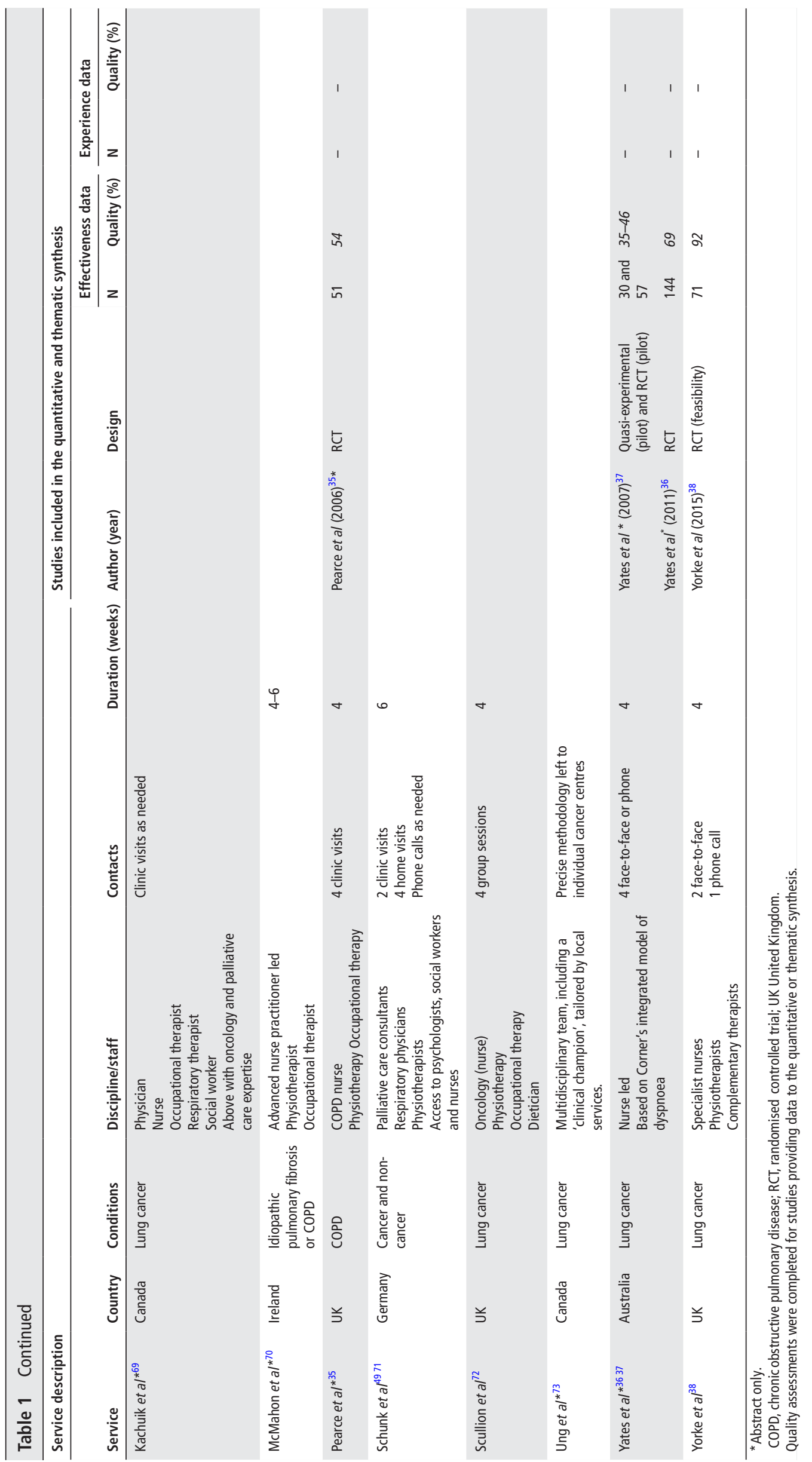

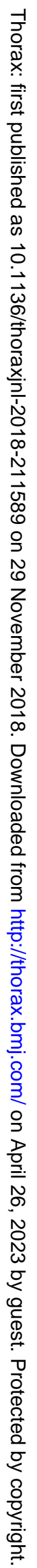




\begin{tabular}{lcl}
\hline \multicolumn{1}{l}{ Table 2 Service components } & \\
\hline Intervention & $\mathrm{n}$ & \multicolumn{1}{l}{ Services* } \\
\hline Information and education & & \\
\hline Education/advice & 9 & 182021384957586973 \\
\hline Nutritional advice/support & 3 & 205872 \\
\hline Sleep hygiene & 2 & 2066 \\
\hline Smoking cessation advice/ & 1 & 20 \\
\hline support & & \\
\hline Written information & 4 & 20214958 \\
\hline Psychosocial support & & \\
\hline Carer/family support & 5 & 1820214973 \\
\hline Psychological support & 12 & 182021353746495859697273 \\
\hline Social support & 7 & 18202135374958 \\
\hline Spiritual support & 1 & 21 \\
\hline Self-management strategies & & \\
\hline Breathing techniques & 14 & $18202135373840464957-596670$ \\
\hline Emergency/crisis planning & 3 & 202149 \\
\hline Exercise plans & 5 & 2021495861 \\
\hline Handheld fan/water spray & 5 & 2021495859 \\
\hline Goal-setting & 4 & 18204959 \\
\hline Pacing & 8 & 2021404649585972 \\
\hline Positioning & 4 & 20214958 \\
\hline Relaxation/calming techniques & 11 & 1820213540464958597072 \\
\hline Other interventions & & \\
\hline Accupressure/TENS & 2 & 3861 \\
\hline Occupational aids & 5 & 2021495861 \\
\hline Pharmacological review & 4 & 20216670 \\
\hline TENS , transcutaneous electrical nerve stimulation. \\
\hline
\end{tabular}

occupational therapists, with involvement from palliative care, respiratory care and oncology. Services used a wide range of interventions (table 2), most commonly breathing techniques, psychological support and relaxation or calming techniques. A minority ( $\leq 2$ of 18 ) included acupressure/transcutaneous electrical nerve stimulation, sleep hygiene advice, spiritual support or smoking cessation interventions.

\section{Effectiveness of holistic breathlessness services}

Twelve studies (11 RCTs ${ }^{18-21} 34-40$ and 1 quasi-experimental design $^{37}$ ) from seven services were included in the quantitative synthesis (table 1). Of these five were designed as pilot/feasibility studies $^{18} 363839$ and seven as effectiveness studies. ${ }^{19-21}$ 34-36 40 Nine studies compared the services to usual care; in one study ${ }^{18}$ the control group were encouraged to talk freely about their breathlessness and disease but not offered training or counselling, and two studies compared one versus three contacts with a service. ${ }^{39} 40$

Nine studies enrolled only patients with cancer, ${ }^{18193436-40}$ two enrolled only patients with non-malignant disease ${ }^{20}$ or $\mathrm{COPD}^{35}$ and one study enrolled patients with any advanced disease. ${ }^{21}$ In total, 979 patients were recruited (range $22^{39}$ to $156^{40}$ ), including 757 (77.3\%) with advanced cancer and 180 (18.4\%) with advanced COPD. The remaining participants $(4.3 \%)$ had other non-malignant diseases including interstitial lung disease or heart failure. A wide variety of outcomes were measured (online supplementary table S1). The most common measures

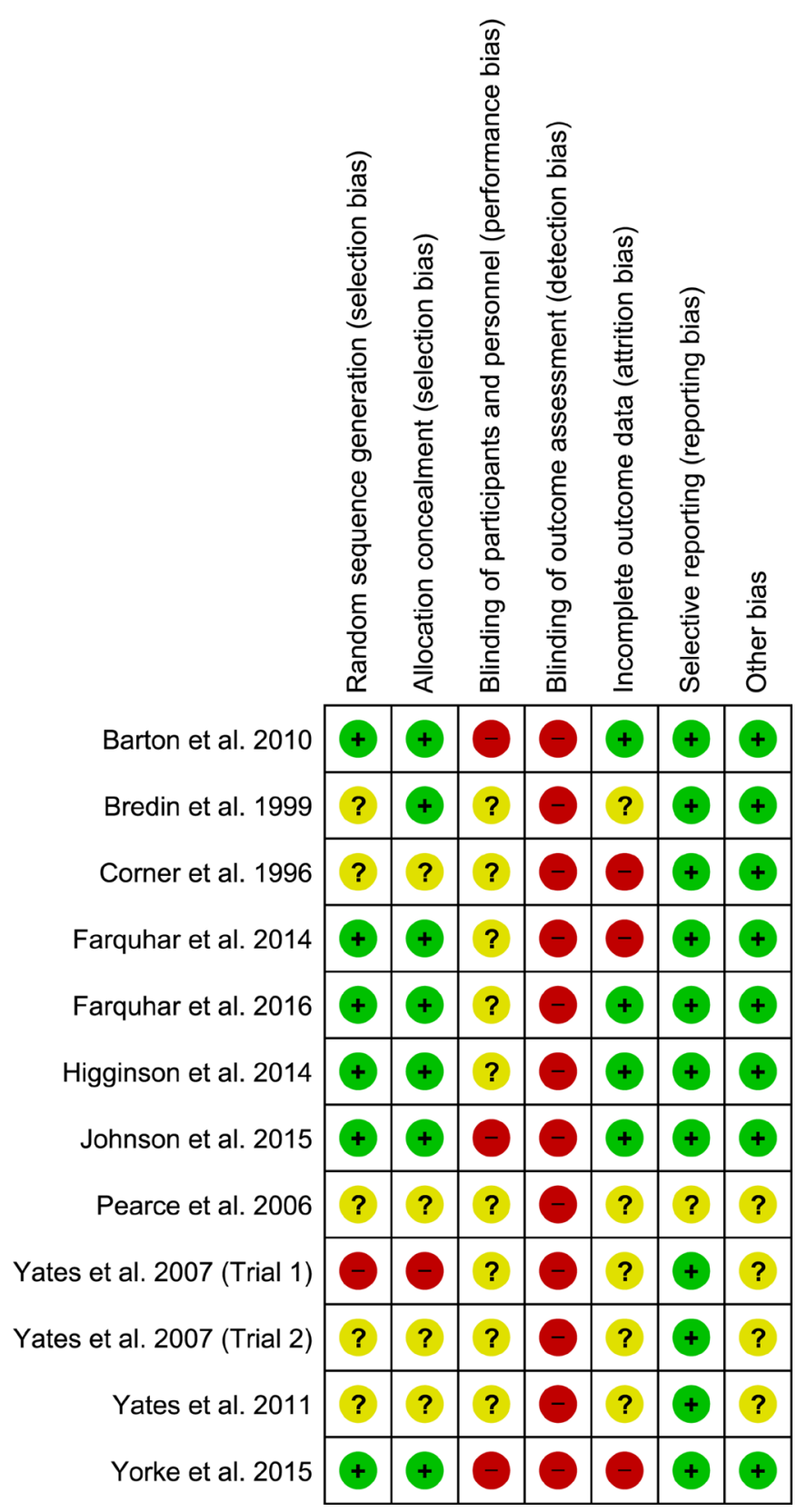

Figure 2 Risk of bias summary.

were breathlessness intensity (10 studies), distress due to breathlessness (10 studies) and anxiety and depression (9 studies). Breathlessness intensity measures varied by type (average/best/ worst), context (at rest/on exertion) and timing (current/past 24 hours/past week).

Quality assessment scores ranged from 35\% to 100\% (median 90.4\%; online supplementary table S2). The lowest scores were for studies where only an abstract was available. ${ }^{35-37}$ Due to the nature of the intervention that prohibits patient blinding and prioritises self-assessed outcomes, all studies were deemed at risk of detection bias and most at risk of performance bias (figure 2). Only three studies reported blinding of investigators. ${ }^{19-21}$

\section{Breathlessness intensity}

Ten studies ${ }^{18} 2134-40$ assessed the severity of breathlessness using one or more of the following measures: visual analogue scale (VAS), Numeric Rating Scale (NRS) or Borg scores (see online 
supplementary table $\mathrm{S} 3$ for details). For 'best breathlessness' two studies using VAS found a greater improvement compared with control (differences in median change 5.7, $\mathrm{p}=0.03^{34}$ and 1.0 , $\mathrm{p}=0.02)^{18}$ and three studies with unspecified measures found a significant intervention effect $(F[2,44]=5.30, p=0.009)^{37}$ or no difference (data not reported). ${ }^{3637}$ For 'worst breathlessness' one study using VAS found a greater improvement compared with control (difference in median change 3.5, $\mathrm{p}=0.05$ ), ${ }^{18}$ whereas no significant differences were found by two studies using NRS (MD $-0.35,95 \% \mathrm{CI}-1.71$ to $1.01, \mathrm{p}=0.61^{21}$; MD $0.41,95 \% \mathrm{CI}$ -0.86 to $1.67, \mathrm{p}=0.53$ ), ${ }^{38}$ one study using VAS (difference in median change $3.8, \mathrm{p}=0.14)^{34}$ and one with an unspecified measure (data not reported). ${ }^{36}$ For 'average breathlessness' one study using an unspecified measure found a greater improvement compared with control (difference in mean change 1.2, ${ }^{36}$ whereas two studies using NRS did not (MD $-0.33,95 \% \mathrm{CI}$ -1.28 to $0.62, \mathrm{p}=0.49^{21}$; MD $0.65,95 \% \mathrm{CI}-0.49$ to 1.80 , $\mathrm{p}=0.26) .{ }^{38}$ One study using NRS found no effect on breathlessness on exertion (MD $-0.7395 \% \mathrm{CI}-1.69$ to $0.22, \mathrm{p}=0.13),{ }^{21}$ and one study using Borg scale ratings for breathlessness at rest and on exertion found no difference between groups (data not reported). ${ }^{35}$ In line with their feasibility study results, ${ }^{39}$ a powered trial comparing one with three service contacts found no significant difference in NRS worst (MD 0.2, 95\% CI -2.31 to $2.97, \mathrm{p}=0.83$ ) or average (MD $0.3,95 \% \mathrm{CI}-2.00$ to 2.62 , $\mathrm{p}=0.79$ ) breathlessness. ${ }^{40}$

\section{Breathlessness affect}

Ten studies ${ }^{18-20} 34$ 36-40 assessed 'distress due to breathlessness' using VAS (range 0-100, higher=worse) or NRS (range 0-10, higher $=$ worse), two as a prespecified primary outcome. ${ }^{19}{ }^{20}$ Of eight studies comparing breathlessness services to usual care, data from five ${ }^{18-203438}$ were pooled in a meta-analysis $(n=324$, figure $3 \mathrm{~A}$ ). Three studies ${ }^{36}{ }^{37}$ reported no significant difference but could not be included as data were not reported. Meta-analysis of those studies reporting data showed significantly lower NRS distress following the intervention compared with control (MD $-2.30,95 \% \mathrm{CI}-4.43$ to $-0.16, \mathrm{p}=0.03$ ). A sensitivity analysis excluding two outlier studies ${ }^{1834}$ resulted in a reduced point estimate of effect and non-significant difference (MD $-0.29,95 \% \mathrm{CI}-1.00$ to $\left.0.43, \mathrm{p}=0.43 ; \mathrm{I}^{2}=0 \%\right)$. One feasibility study ${ }^{39}$ and one randomised trial ${ }^{40}$ testing service variations found no difference on NRS 'coping with breathlessness' (MD $-1.7,95 \% \mathrm{CI}-4.27$ to $0.90, \mathrm{p}=0.20)^{40}$ and significantly higher NRS distress due to breathlessness following three sessions versus one session (MD 3.9, 95\% CI 0.98 to 6.91, $\mathrm{p}=0.01){ }^{40}$

Four studies ${ }^{19-2135}$ assessed 'mastery over breathlessness' using the Chronic Respiratory Questionnaire mastery domain; two as a primary outcome. ${ }^{2135}$ A meta-analysis of these data $(n=259$, figure $3 \mathrm{~B}$ ) showed a statistically non-significant increase in mastery (range $1-7$, higher=better) favouring the intervention (MD $0.23,95 \% \mathrm{CI}-0.10$ to $0.55, \mathrm{p}=0.17$ ). A sensitivity analysis excluding one study ${ }^{35}$ deemed at high risk of bias increased the point estimate of effect (MD 0.30, 95\% CI -0.06 to 0.66, $\mathrm{p}=0.11)$. One study found significantly lower mastery scores following three compared with one service contact (MD -0.6 , $95 \% \mathrm{CI}-1.06$ to $-0.11, \mathrm{p}=0.02){ }^{40}$

One further study found improved dyspnoea- 12 (range 0-36; higher $=$ worse) scores following intervention as compared with control (MD 5.19, 95\% CI 0.62 to $9.75, \mathrm{p}=0.026) .{ }^{38}$

\section{Psychological outcomes}

Seven studies assessed anxiety and depression using the Hospital Anxiety and Depression Scale (HADS). ${ }^{18-21} 343638$ Data from these seven studies ( $\mathrm{n}=552$, figure $3 \mathrm{C}$ ) showed a statistically non-significant reduction in anxiety scores (range 0-21; high$\mathrm{er}=$ worse $)$ favouring the intervention $(\mathrm{MD}-1.59,95 \% \mathrm{CI}$ -3.22 to $0.05, \mathrm{p}=0.06$ ). Sensitivity analysis excluding one study ${ }^{36}$ deemed at high risk of bias increased the point estimate $(-1.85,95 \% \mathrm{CI}-3.76$ to $0.06, \mathrm{p}=0.06)$. Sensitivity analysis removing one outlier study ${ }^{34}$ resulted in a reduced point estimate but statistically significant group difference (MD $-0.66,-1.23$ to $\left.-0.10, \mathrm{p}=0.02 ; \mathrm{I}^{2}=0 \%\right)$. No statistical differences in anxiety were reported when comparing one and three contacts. ${ }^{39} 40$

For depression, one study ${ }^{37}$ reporting no difference between groups could not be included in the meta-analysis as data were not provided. Meta-analysis using six remaining studies $(n=408$, figure 3D) showed reduced depression scores (range 0-21, higher $=$ worse $)$ favouring the intervention $(\mathrm{MD}-1.67,95 \% \mathrm{CI}$ -2.52 to $-0.81, \mathrm{p}<0.001)$. No statistical differences in depression were reported when comparing one and three contacts. ${ }^{39} 40$

Three further studies reported no significant differences between the intervention and control groups in 'psychological symptoms'; two using an unspecified measure (data not reported) ${ }^{3637}$ and one using the Rotterdam Symptom Checklist (range 7-28, higher $=$ worse; difference in median change -8 , $\mathrm{p}=0.21) .{ }^{34}$ One study comparing one session with three sessions found no significant difference on CRQ emotion scores (MD $-0.09,95 \% \mathrm{CI}-0.54$ to $0.36, \mathrm{p}=0.69){ }^{40}$

\section{Physical function, health status and survival}

Five studies ${ }^{18-21} 34$ assessed physical function. Two studies found greater improvements following intervention compared with control using the Functional Capacity Scale (range 0-14; higher=better; MD for change $1.25, \mathrm{p}<0.02)^{18}$ and WHO Performance Scale (range 0-5, higher $=$ worse; difference in median change $-2, p=0.02),{ }^{34}$ respectively. Three studies observed no difference in functional outcomes between groups assessed using either the London Chest Activities of Daily Living Scale ${ }^{21}$ (MD $-5,95 \% \mathrm{CI}-12.22$ to $1.02, \mathrm{p}=0.10$ ) or patient-reported number of times out of house ${ }^{19} 20$ (data not reported).

Seven studies ${ }^{19-21} 34353840$ included a measure of health status or quality of life. No significant differences were found between groups across the CRQ dyspnoea domain ${ }^{19-21} 35$ (including the comparison between one and three sessions ${ }^{40}$ ) or total score, ${ }^{21}$ EuroQol-5D index ${ }^{21}$ or VAS, ${ }^{21} 38$ and the Rotterdam Symptom Scale quality-of-life domain. ${ }^{34}$ Due to heterogenous measures, change from baseline and post-intervention scores, and cases of non-normally distributed data, we decided against meta-analysis for these outcomes.

Two studies reported survival data. ${ }^{2134}$ One found a significant difference in survival (generalised Wilcoxon score 3.9, $\mathrm{p}=0.048$ ) in favour of the intervention. ${ }^{21}$ Subgroup analysis found the difference was driven by participants with non-cancer diagnoses. The remaining study, enrolling only patients with cancer, found no difference in survival across groups (data not reported). ${ }^{34}$

\section{Economic evaluation}

One service did not increase formal care costs compared with usual care (mean (SD) $£ 2911$ ( $£ 2729)$ vs $£ 3709$ ( $£ 4484)$; incremental QALY gain 0.092 (95\% CI -0.23 to 0.04)). ${ }^{21}$ Another service ${ }^{1920}$ was more cost-effective than usual care for patients with cancer (total costs $£ 354$ lower $(95 \% \mathrm{CI}-£ 1020$ to $£ 246$ ); incremental QALY-gain 0.0002 (95\% CI -0.001 to 0.002$)$ ), ${ }^{19}$ 


\subsection{NRS Distress due to breathlessness}

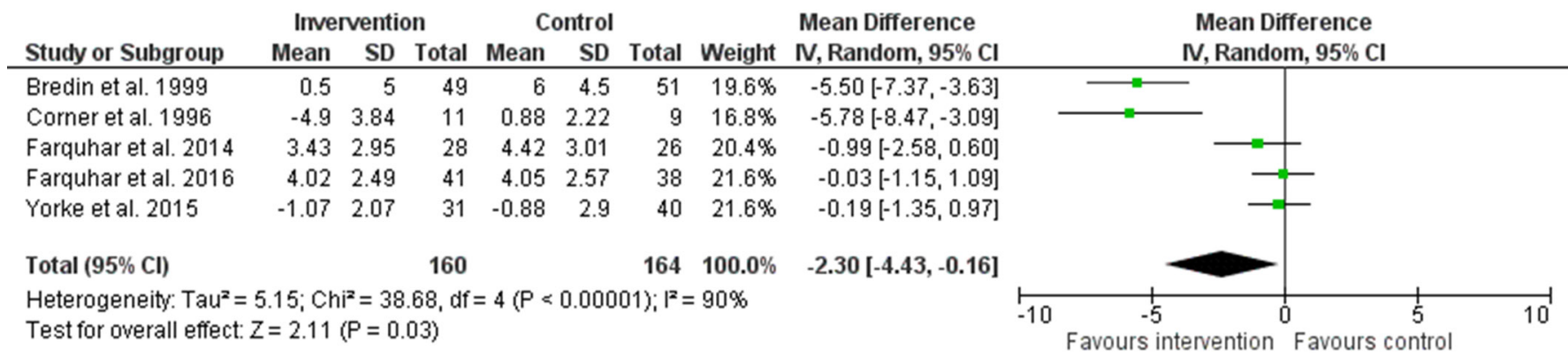

\subsection{CRQ Breathlessness Mastery}

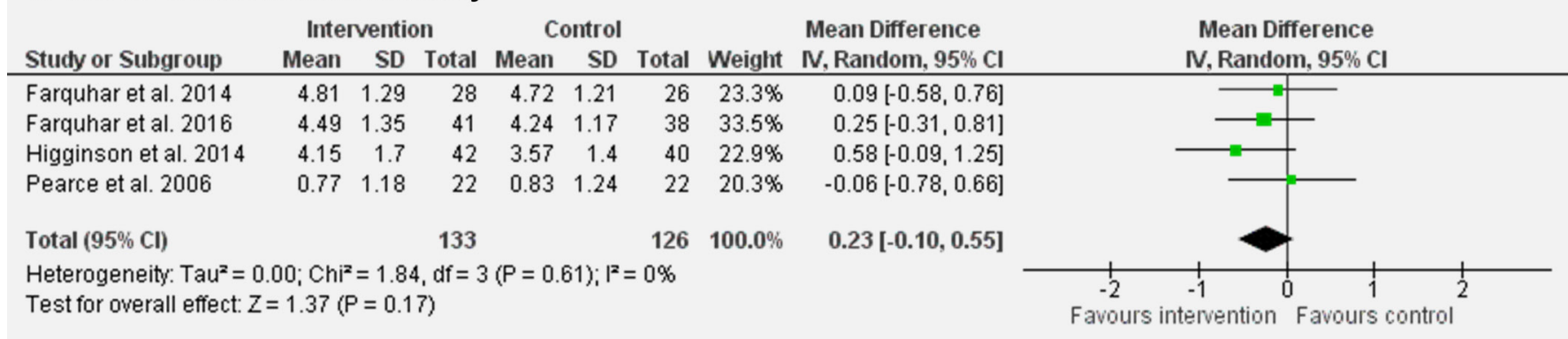

\subsection{HADS Anxiety}

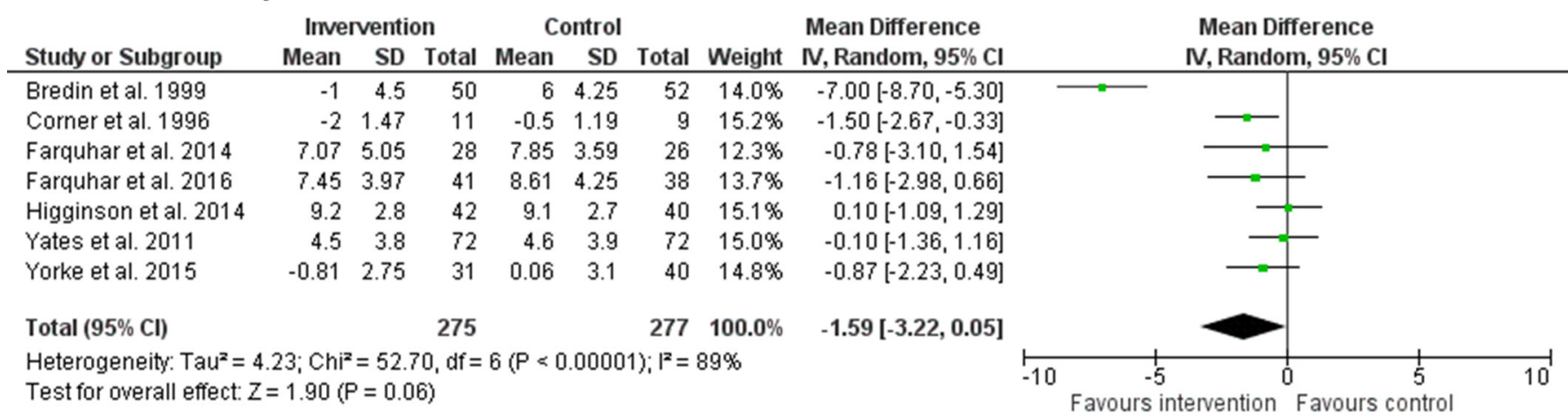

\subsection{HADS Depression}

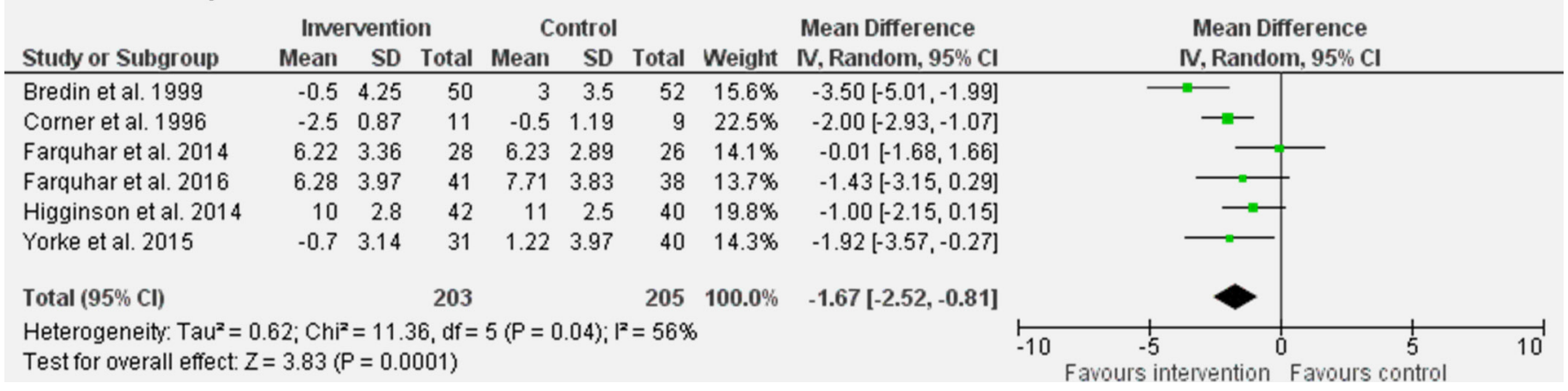

Figure 3 Meta-analyses. HADS, Hospital Anxiety and Depression Scale; NRS, Numeric Rating Scale.

but not non-cancer conditions (total costs $£ 799$ higher (95\% CI $-£ 237$ to $£ 1904)$; 0.003 QALY gain $(95 \% \mathrm{CI}-0.001$ to 0.007$)) .^{20}$ A third service enrolling patients with cancer found a non-significant reduction in QALYs following three sessions compared with one session (MD $-0.006(95 \%$ CIs -0.018 to 0.006$)) .{ }^{40}$

\section{Experiences of holistic breathlessness services}

Twelve articles ${ }^{18-2141-48}$ reporting experience data from five separate services were included in the qualitative synthesis (table 1).
These included six mixed-method ${ }^{18-21} 434647$ and five qualitative studies. ${ }^{41} 42444548$ Most data were from patient and/or carer interviews ${ }^{18-2141-45} 48$; one used therapists notes, ${ }^{46}$ and one used free-text responses to a postal survey. ${ }^{47}$ Data represented views of 167 patients (53.9\% with cancer) and up to 49 carers. Quality assessment scores ranged from $40 \%$ to $85 \%$ (median $70 \%$; online supplementary table S4). Common limitations included lack of reflexivity, not using verification procedures to establish credibility and unclear reporting of the analytic methods. 
Three themes were identified: valued characteristics, perceived outcomes and challenges to services.

Patients valued the education and information sharing included in the services, particularly to help them understand their breathlessness, legitimise the treatments being suggested, and provide resources to refer to in future crises (Box 2). The treatments themselves (breathing techniques, pacing, positioning, relaxation, handheld fan) were praised for their simplicity, portability and perceived effectiveness. The psychosocial support received through the services was highly valued, providing opportunities for participants to have their experiences listened to and acknowledged, receive support and reassurance, and discuss problems beyond their breathlessness. Participants appreciated when carers were involved, both to support them as individuals and in caring for the patient. Overwhelmingly, recipients commented on the qualities of the staff providing services, whom they deemed experts in not only managing breathlessness, but in person-centred care and treating participants with respect and dignity.

In line with quantitative findings, perceived outcomes were mostly psychological, including increased understanding and self-efficacy, and feeling more 'in control', less isolated, or less distressed (Box 2). Some recipients also reported their breathing feeling easier and more controlled. Others felt the sensation of breathlessness was unchanged, but their reaction to it had changed. Participants reported being more able to maintain and/ or increase their daily activity, and successfully self-manage.

Two potential challenges for services were identified (Box 2). First, respondents' accounts showed the importance of motivation to self-manage in the success of the interventions, yet this was difficult if benefits were not quickly achieved. Second, some participants had low expectations of the services or the treatments offered, which at times resulted in a reluctance to engage.

\section{DISCUSSION}

This review synthesised quantitative and qualitative evidence to provide a detailed understanding of holistic services for people with advanced disease and breathlessness that persists despite optimal treatment of the underlying disease. Despite wide variation across health service models, we found evidence suggesting an effect on the affective domain of breathlessness, and on psychological outcomes of anxiety and depression. Services were highly valued by patients and carers, who appreciated the education to help them understand their breathlessness, the provision of useful self-management interventions and the provision of expert dignified care which centred on the person. There was however no effect on overall health status or quality of life using varied generic measures, and mixed evidence around any effect on physical function.

To our knowledge, this is the first systematic review in this field; a recent narrative review described some common service features, but focused on an emerging service in Munich that contributed to our data. ${ }^{49}$ The bias of effect towards psychological health outcomes is concordant with the primary focus of these services to support living with breathlessness rather than taking the symptom away. The effect on depression, which in cases arose from preventing its onset within usual care, ${ }^{21} 34$ may have been achieved through expert management of breathlessness and concurrent symptoms, but also through receipt of holistic care that prioritised active listening and putting the person before their disease. The effect sizes observed (point estimates: distress: -0.57 , mastery: 0.18 , anxiety -0.45 , depression: -0.55$)$ are larger than those achieved with psychological
Box 2 Example quotes for themes derived from qualitative synthesis

\section{Valued service characteristics}

- Education and information sharing: 'When I'm having problems I go back and read it to see if I am doing the right thing. I find that very, very helpful.' (Man, ILD) ${ }^{21}$

- Caring and expert service providers: 'Would you like a cup a tea [...] it's just human to human situation. But that environment makes you: you are in the right place, you know. There is no guessing going on, [...]. You are gonna get the best of their mind.' (P01043, man with COPD) ${ }^{45}$

- Involving carers: 'Knowing now that he won't die in one of these sort of situations, so that certainly helped me, and it certainly helped me to realise that, you know, I can probably help him to calm down. So yes, as a carer I think it was a help.' $(038 \mathrm{t} 3 \mathrm{c})^{20}$

- Psychological support: 'I was able to discuss my personal feelings, that you don't talk to your family about so not to worry them.' (69-year-old woman with COPD) ${ }^{47}$

- Simple, portable and effective tools: 'To put my hand on my tummy ... puff puff puff ... and do that, and you know, it's amazing really, it sounds so pathetic when you say something ... It is simple, it's not a thing you'd think of doing.' (530t3pc) ${ }^{19}$

\section{Perceived outcomes}

Affective distress

- Increased self-efficacy: 'They were increasing activity and functional levels by using breathing techniques and exploiting the confidence these gave them.' (Researcher comments) ${ }^{18}$

- Feeling more 'in control': 'The blissful thing is, like I've said is, you can control your breathing, if you get a bad spell you can work your way through it.' (Man, ILD) ${ }^{21}$

- Feeling less isolated: 'At this time you're down and lonely anyway so having someone there for support is important.' $(04 \mathrm{M})^{48}$

- Increased understanding: 'I was thrilled to bits to be able to be getting some knowledge of what my complaint was all about ... that they're doing something about it.' (P04) ${ }^{41}$

- Reduced distress: 'Learning to relax and not get so stressed out, I mean I still get the old panic now and again when I've really put myself out [...], but most of the time I deal with it quite easily.' (Patient with cancer) ${ }^{44}$

Sensory-perceptual experience

- Perceived impact on what breathing feels like: 'It helped me to learn to relax, learn to breathe in a more controlled way.' (54-year-old man with COPD) ${ }^{47}$

Symptom impact or burden

- Improved daily activities: 'Went up and down [the stairs] with very little increase in respiratory rate. Flung her arms around my neck and said "I never thought I would be able to do that again".' (Staff comment 11) ${ }^{46}$

- Increased self-management: 'I would get into a panic when I was breathless, but now I can sit down use my fan, wet my face, read my laminate (breathlessness poem) and I calm down.' (Woman with COPD) ${ }^{21}$

\section{Challenges to services}

- Motivation: 'She gave me a fan and told me to, you know, put it on ... and then blow out. I do try to do it, but I get so out of breath doing it. I give up.' (Case 013$)^{43}$ 


\section{Box 2 Continued}

- Expectations: 'Hoping that something would help me but a little bit cynical as well ... I didn't see how anything could help improve it.' $(03 \mathrm{M})^{48}$

therapies, self-management programmes and more comparable to pulmonary rehabilitation, ${ }^{50} 51$ despite the different interventional approach. While few measurable effects were identified for physical function and quality of life, we feel it important to acknowledge the qualitative data that captured participants' feelings of expanding horizons, including being able to maintain daily activities and tasks. The diverse nature of patient-reported improvements in physical function may be difficult to capture using standardised measures, and individualised measures (eg, goal attainment scaling ${ }^{52}$ ) could have more utility in this setting.

We intentionally made no attempt to compare with pulmonary rehabilitation, although in chronic respiratory disease the interface between the two service models must be addressed. In no respect do we view holistic breathlessness services as a replacement for pulmonary rehabilitation, which is a highly effective and underused intervention. ${ }^{14}$ These services may however act as a next step for people who remain highly symptomatic despite completing pulmonary rehabilitation, as a bridge for people limited by chronic breathlessness but who decline pulmonary rehabilitation (which may include people who are post-admission for an acute exacerbation), and/or as an adjunct for patients whose goals relate to psychosocial health. Homebased pulmonary rehabilitation provision overcomes some issues with transportation and improves reach. ${ }^{53}$ However, holistic breathlessness service services may provide an additional opportunity for health gains in people unable to complete programmes with a major exercise component, particularly where breathlessness limits people from exercising to an intensity associated with a training response. ${ }^{6} 1655$ The inclusion of palliative care may also be helpful for this population, who have distressing physical and psychological symptoms, often limited understanding of their disease, and infrequently discuss end-of-life issues in routine clinical care. ${ }^{24}$ Although international guidelines advocate for early integration of palliative care in chronic disease, ${ }^{22} 23$ the unpredictable course of many respiratory conditions, including COPD, and the difficulty of predicting survival are barriers to timely palliative care referral and receipt. A symptom-triggered approach should reach more people likely to benefit than current approaches based on prognostication. ${ }^{24}$ For services already well-aligned with palliative care, adoption of the core therapeutic components for breathlessness management into existing practice may suffice.

The heterogeneity of service models with respect to staffing, structure, content and target populations is an important finding. While some shared characteristics were identified (Box 3), further work is required to determine the most effective components, and which recipients gain most benefit. This includes determining optimal service duration, particularly as one trial found better outcomes for distress due to breathlessness and mastery after one session versus three (hypothesising that one session increased self-efficacy and reduced logistic challenges of multiple clinic visits). The literature is small but increasing, and new data from services identified with no published outcomes can be expected. Use of consistent measures may permit meta-regression, or responder analysis using individual level data to identify service and patient characteristics related to better outcomes.
Box 3 Typical composition of a holistic breathlessness service

Intended users

- People with advanced disease and chronic breathlessness despite optimal disease management, and their informal carers

Philosophy

- Optimising the person's ability to live with and self-manage breathlessness, with a focus on the person before their disease

Staffing

- Multidisciplinary team of experts in breathlessness and dignified care

Setting

- Mixture of face-to-face support in clinics and/or at home, and phone support

Interventions

- Information and education, psychological support, selfmanagement strategies and other appropriate interventions

An alternative approach is to use discrete choice experiments to identify which components would be prioritised and preferred by patients and carers, particularly in resource-limited settings. Findings would inform future services as appropriate, but also adoption of the most effective characteristics into existing services upstream. Increased consideration of cost effectiveness is also warranted.

Strengths of this work include a registered protocol, and a systematic and comprehensive search across multiple databases, inclusive of grey literature, with no exclusions by publication year or language. Eligibility and quality assessment was conducted independently by two authors, and multiple stakeholders (researchers, clinicians, service user representatives) contributed to the analysis and interpretation of these data. The review also has some limitations. First, the meta-analyses included data from services shown to vary in structure, delivery and recipients. We completed sensitivity analyses in response to any clinical heterogeneity, but the overall dataset was moderate in size and sensitivity analysis compromised the precision of our effect size estimates. These estimates may be inflated by lack of blinding of study personnel in some instances, and disappointment effects in control groups where a fast-track design was not used. Moreover, although we did not assess for statistical evidence of publication bias, there was clear evidence of selective reporting where study authors did not provide data for statistically non-significant findings. Some of our estimates do not include these data, and caution should be applied in these instances. There were also challenges with inconsistent use of, and unclear reporting of, outcome measures, which sometimes precluded meta-analysis (eg, breathlessness intensity). For the qualitative synthesis, included data were drawn from published studies or abstracts. This created an additional layer of abstraction, although also allowed synthesis of study authors' interpretations as we did not limit data extraction to direct quotations. Qualitative data were predominantly drawn from two UK services, ${ }^{19-21}$ and patients who had fully engaged with the services. Less is understood about experiences of these services internationally, of carers, and of those who dropped out and perhaps might report less benefit. Finally, we limited the review to studies in people with advanced disease, which reflects key studies in the current evidence base. We acknowledge that 
service access based on disease severity may however not serve those patients with distressing breathlessness but in early stages of disease by traditional markers, for example, moderate airflow obstruction or potentially resectable thoracic cancer. As such, we advocate access to these services primarily based on the presence of breathlessness, accepting the empirical data presented does not extend to some groups.

In conclusion, holistic services for chronic breathlessness in people with advanced disease overall demonstrate positive effects on patient distress due to breathlessness and psychological health. Services are heterogonous in their content and delivery, but are highly valued by patients and families, who appreciate tailored education around breathlessness, provision of simple, portable self-management interventions and expert staff providing person-centred, dignified care. Chronic or distressing breathlessness can serve as an appropriate referral indicator for timely referral and receipt of palliative care, especially in non-cancer conditions where prognostication causes delays. Further work should test and understand the most effective service configurations and how these can be integrated into existing healthcare systems.

Correction notice This article has been corrected since it was published Online First. Sophie Miller and Lisa Jane Brighton were not originally listed joint first authors.

Acknowledgements The authors thank their team of service user representatives who have provided their reflections and feedback throughout the conduct of the review. Thank you to Margaret Ogden and Charlie Reilly for providing their feedback on the qualitative analysis.

Contributors MM and IJG designed the study, obtained funding and take overall responsibility for its content. LJB and SM conducted the searches and data extraction, MM, LJB, SM and IJH assessed study eligibility, and MM and LJB assessed study quality. MM, LJB, SM, MF and DY analysed and interpreted the data. All authors contributed to the manuscript and approved the final version.

Funding This review was conducted as part of a project funded by an NIHR Health Services and Delivery Research Grant (NIHR HSDR 16/02/18). MM, GW and IJH are supported by the NIHR Collaboration for Leadership in Applied Health Research and Care (CLAHRC) South London and Cicely Saunders International; MM is supported by an NIHR Career Development Fellowship (CDF-2017-009); WM is supported by the NIHR CLAHRC Northwest London, and IJH holds an NIHR Emeritus Senior Investigator Award.

\section{Competing interests None declared.}

Patient consent Not required.

Provenance and peer review Not commissioned; externally peer reviewed.

Data sharing statement Requests for additional data should be addressed to the corresponding author.

Open access This is an open access article distributed in accordance with the Creative Commons Attribution 4.0 Unported (CC BY 4.0) license, which permits others to copy, redistribute, remix, transform and build upon this work for any purpose, provided the original work is properly cited, a link to the licence is given, and indication of whether changes were made. See: https://creativecommons.org/ licenses/by/4.0/.

\section{REFERENCES}

1 Solano JP, Gomes B, Higginson IJ. A comparison of symptom prevalence in far advanced cancer, AIDS, heart disease, chronic obstructive pulmonary disease and renal disease. J Pain Symptom Manage 2006;31:58-69.

2 Higginson IJ, Hart S, Silber E, et al. Symptom prevalence and severity in people severely affected by multiple sclerosis. J Palliat Care 2006;22:158-65.

3 Etkind $\mathrm{SN}$, Bone $\mathrm{AE}$, Gomes $\mathrm{B}$, et al. How many people will need palliative care in 2040? Past trends, future projections and implications for services. BMC Med 2017;15:102.

4 Seow H, Barbera L, Sutradhar R, et al. Trajectory of performance status and symptom scores for patients with cancer during the last six months of life. J Clin Oncol 2011;29:1151-8.

5 Johnson MJ, Yorke J, Hansen-Flaschen J, et al. Towards an expert consensus to delineate a clinical syndrome of chronic breathlessness. Eur Respir J 2017;49:1602277
6 Gysels MH, Higginson IJ. The lived experience of breathlessness and its implications for care: a qualitative comparison in cancer, COPD, heart failure and MND. BMC Palliat Care 2011;10:15.

7 Farquhar M. Carers and breathlessness. Curr Opin Support Palliat Care 2017;11:165-73

8 Bausewein C, Booth S, Gysels M, et al. Understanding breathlessness: cross-sectional comparison of symptom burden and palliative care needs in chronic obstructive pulmonary disease and cancer. J Palliat Med 2010;13:1109-18.

9 Janssen DJ, Spruit MA, Uszko-Lencer NH, et al. Symptoms, comorbidities, and health care in advanced chronic obstructive pulmonary disease or chronic heart failure. J Palliat Med 2011;14:735-43.

10 Ekström M, Bajwah S, Bland JM, et al. One evidence base; three stories: do opioids relieve chronic breathlessness? Thorax 2018;73:88-90.

11 Barnes H, McDonald J, Smallwood N, et al. Opioids for the palliation of refractory breathlessness in adults with advanced disease and terminal illness. Cochrane Database Syst Rev 2016;3:CD011008.

12 Simon ST, Higginson IJ, Booth S, et al. Benzodiazepines for the relief of breathlessness in advanced malignant and non-malignant diseases in adults. Cochrane Database Syst Rev 2016:10:CD007354.

13 Spathis A, Booth S, Moffat C, et al. The breathing, thinking, functioning clinical model: a proposal to facilitate evidence-based breathlessness management in chronic respiratory disease. NPJ Prim Care Respir Med 2017;27:27

14 McCarthy B, Casey D, Devane D, et al. Pulmonary rehabilitation for chronic obstructive pulmonary disease. Cochrane Database Syst Rev 2015;2:Cd003793.

15 Taylor RS, Sagar VA, Davies EJ, et al. Exercise-based rehabilitation for heart failure Cochrane Database Syst Rev 2014;7.

16 Brown TM, Hernandez AF, Bittner V, et al. Predictors of cardiac rehabilitation referral in coronary artery disease patients: findings from the American Heart Association's Get With The Guidelines Program. J Am Coll Cardiol 2009:54:515-21.

17 Maddocks M, Kon SS, Canavan JL, et al. Physical frailty and pulmonary rehabilitation in COPD: a prospective cohort study. Thorax 2016;71:988-95.

18 Corner J, Plant $H_{\text {, A }}$ Hern R, et al. Non-pharmacological intervention for breathlessness in lung cancer. Palliat Med 1996;10:299-305.

19 Farquhar MC, Prevost AT, McCrone P, et al. Is a specialist breathlessness service more effective and cost-effective for patients with advanced cancer and their carers than standard care? Findings of a mixed-method randomised controlled trial. BMC Med 2014:12:194.

20 Farquhar MC, Prevost AT, McCrone P, et al. The clinical and cost effectiveness of a Breathlessness Intervention Service for patients with advanced non-malignant disease and their informal carers: mixed findings of a mixed method randomised controlled trial. Trials 2016:17:185

21 Higginson IJ, Bausewein C, Reilly CC, et al. An integrated palliative and respiratory care service for patients with advanced disease and refractory breathlessness: a randomised controlled trial. Lancet Respir Med 2014;2:979-87.

22 Ferrell BR, Temel JS, Temin S, et al. Integration of palliative care into standard oncology care: american society of clinical oncology clinical practice guideline update. J Clin Oncol 2017:35:96-112.

23 Vogelmeier CF, Criner GJ, Martinez FJ, et al. Global strategy for the diagnosis, management, and prevention of chronic obstructive lung disease 2017 report. GOLD executive summary. Am J Respir Crit Care Med 2017;195:557-82.

24 Maddocks M, Lovell N, Booth S, et al. Palliative care and management of troublesome symptoms for people with chronic obstructive pulmonary disease. Lancet 2017:390:988-1002.

25 Moher D, Liberati A, Tetzlaff J, et al. Preferred reporting items for systematic reviews and meta-analyses: the PRISMA statement. Ann Intern Med 2009;151:264-9.

26 Maddocks M, Higginson IJ, Farquhar M, et al. Protocol: Holistic services for refractory breathlessness in advanced malignant and non-malignant disease. 2017. http://www. crd.york.ac.uk/PROSPERO/display_record.php?ID=CRD42017057508 (Accessed 26 Jan 2018)

27 Parshall MB, Schwartzstein RM, Adams L, et al. An official American Thoracic Society statement: update on the mechanisms, assessment, and management of dyspnea. Am J Respir Crit Care Med 2012;185:435-52.

28 Kmet LM, Lee RC, Cook LS. Standard quality assessment criteria for evaluating primary research papers from a variety of fields. 2004. https://www.biomedcentral.com/ content/supplementary/1471-2393-14-52-s2.pdf (Accessed 02 Jun 2017).

29 Higgins JPT, Altman DG, Gotzsche PC, et al. The Cochrane Collaboration's tool for assessing risk of bias in randomised trials. BMJ 2011;343:d5928.

30 QSR International Pty Ltd. NVivo qualitative data analysis software, version 10, 2012.

31 Higgins J, Green S. Cochrane handbook for systematic reviews of interventions 5.1.0: 9.5.2 identifying and measuring heterogeneity. 2011. http://handbook-5-1.cochrane. org/ (accessed 19 Jan 2017).

32 Higgins J, Green S. Cochrane handbook for systematic reviews of interventions 5.1.0: 10.4 detecting reporting biases. 2011. http://handbook-5-1.cochrane.org/ (accessed 19 Jan 2017).

33 Thomas J, Harden A. Methods for the thematic synthesis of qualitative research in systematic reviews. BMC Med Res Methodol 2008;8:45. 
34 Bredin M, Corner J, Krishnasamy M, et al. Multicentre randomised controlled trial of nursing intervention for breathlessness in patients with lung cancer. BMJ 1999;318:901-4.

35 Pearce L, MacLeod V, Baker A, et al. Randomised controlled trial of nurse-led breathlessness intervention to improve the management of breathlessness in patients with chronic obstructive pulmonary disease at a district general hospital. Thorax 2006;61:॥82-4.

36 Yates $\mathrm{P}$, Hardy J, Kwun $\mathrm{F}$, et al. A randomised controlled trial of a nonpharmacological intervention for dyspnoea. Asia-Pacific Journal of Clinical Oncology 2011;7:82.

37 Yates P, White E, Skerman H. Evaluating alternate approaches for delivering nonpharmacological interventions for dyspnea in patients with lung cancer.Oncology Nursing Society 32nd Annual Congress, April 24-27, 2007, Las Vegas, NV. Oncology Nursing Forum 2007;34:551-51.

38 Yorke J, Lloyd-Williams M, Smith J, et al. Management of the respiratory distress symptom cluster in lung cancer: a randomised controlled feasibility trial. Supportive Care in Cancer 2015;23:3373-84.

39 Barton R, English A, Nabb S, et al. A randomised trial of high vs low intensity training in breathing techniques for breathless patients with malignant lung disease: a feasibility study. Lung Cancer 2010;70:313-9.

40 Johnson MJ, Kanaan M, Richardson G, et al. A randomised controlled trial of three or one breathing technique training sessions for breathlessness in people with malignant lung disease. BMC Med 2015;13:213.

41 Booth S, Farquhar M, Gysels $M$, et al. The impact of a breathlessness intervention service (BIS) on the lives of patients with intractable dyspnea: a qualitative phase 1 study. Palliat Support Care 2006:4:287-93.

42 Farquhar M, Higginson IJ, Booth S. Modelling the carer support component of a complex intervention for breathlessness in advanced disease. Palliative Medicine 2010;24:S167-S68.

43 Farquhar M, Higginson IJ, Fagan $\mathrm{P}$, et al. Results of a pilot investigation into a complex intervention for breathlessness in advanced chronic obstructive pulmonary disease (COPD): brief report. Palliat Support Care 2010;8:143-9.

44 Gysels M, Reilly CC, Jolley CJ, et al. How does a new breathlessness support service affect patients? Eur Respir J 2015;46:1515-8.

45 Gysels M, Reilly CC, Jolley CJ, et al. Dignity through integrated symptom management: lessons from the breathlessness support service. J Pain Symptom Manage 2016:52:515-24.

46 Hately J, Laurence V, Scott A, et al. Breathlessness clinics within specialist palliative care settings can improve the quality of life and functional capacity of patients with lung cancer. Palliat Med 2003;17:410-7.

47 Reilly CC, Bausewein C, Pannell C, et al. Patients' experiences of a new integrated breathlessness support service for patients with refractory breathlessness: Results of a postal survey. Palliat Med 2016;30:313-22.

48 Wood H, Connors S, Dogan S, et al. Individual experiences and impacts of a physiotherapist-led, non-pharmacological breathlessness programme for patients with intrathoracic malignancy: a qualitative study. Palliat Med 2013;27:499-507.

49 Bausewein C, Schunk M, Schumacher P, et al. Breathlessness services as a new model of support for patients with respiratory disease. Chron Respir Dis 2018;15.

50 Griffiths TL, Burr ML, Campbell IA, et al. Results at 1 year of outpatient multidisciplinary pulmonary rehabilitation: a randomised controlled trial. Lancet 2000;355:362-8.

51 Coventry PA, Bower P, Keyworth C, et al. The effect of complex interventions on depression and anxiety in chronic obstructive pulmonary disease: systematic review and meta-analysis. PLoS One 2013;8:e60532.

52 Turner-Stokes L. Goal attainment scaling (GAS) in rehabilitation: a practical guide. Clin Rehabil 2009;23:362-70.
53 Holland AE, Mahal A, Hill CJ, et al. Home-based rehabilitation for COPD using minimal resources: a randomised, controlled equivalence trial. Thorax 2017:72:57-65.

54 Horton EJ, Mitchell KE, Johnson-Warrington V, et al. Comparison of a structured home-based rehabilitation programme with conventional supervised pulmonary rehabilitation: a randomised non-inferiority trial. Thorax 2018;73:29-36

55 Wedzicha JA, Bestall JC, Garrod R, et al. Randomized controlled trial of pulmonary rehabilitation in severe chronic obstructive pulmonary disease patients, stratified with the MRC dyspnoea scale. Eur Respir J 1998;12:363-9.

56 Endnote Version x7. USA: Thomdon Reuters, 2013.

57 Ahmadi L, Das Gupta T, Lilien T, et al. Breathe easy: a pilot project for dyspnea management in lung cancer patients. Journal of Thoracic Oncology 2011;6(SUPPL. 2):S367-\$68.

58 Chan WL, Ng CW, Lee C, et al. Effective management of breathlessness in advanced cancer patients with a program-based, multidisciplinary approach: the "SOB Program" in Hong Kong. J Pain Symptom Manage 2016:51:623-7.

59 Connors S, Graham S, Peel T. An evaluation of a physiotherapy led nonpharmacological breathlessness programme for patients with intrathoracic malignancy. Palliat Med 2007;21:285-7.

60 Bredin M. Multicentre randomized controlled trial of a nursing intervention for breathlessness in patients with lung cancer: update of study progress. European Journal of Oncology Nursing 1998;2:176-7.

61 Douglas L, English A, Obita GP, et al. P229 Breath-taking outcomes: evaluation of a specialist breathlessness clinic. Thorax 2016;71:A211.1-A211.

62 Farquhar MC, Higginson IJ, Fagan P, et al. The feasibility of a single-blinded fast-track pragmatic randomised controlled trial of a complex intervention for breathlessness in advanced disease. BMC Palliat Care 2009;8:9.

63 Booth S, Moffat C, Farquhar M, et al. Developing a breathlessness intervention service for patients with palliative and supportive care needs, irrespective of diagnosis. J Palliat Care 2011;27:28-36.

64 Farquhar MC, Prevost AT, McCrone P, et al. Study protocol: phase III single-blinded fast-track pragmatic randomised controlled trial of a complex intervention for breathlessness in advanced disease. Trials 2011;12:130.

65 Booth S. Cambridge Breathlessness Intervention Service (CBIS). Progress in Palliative Care 2013:21:224-8.

66 Goffin JR, Kerigan A, Russell SA, et al. Assessment of a dyspnea clinic for patients with thoracic malignancies. Journal of Clinical Oncology 2014;32.

67 Bausewein C, Jolley C, Moxham J, et al. Feasibility of a new out-patient breathlessness support service. Palliative Medicine 2012;26:653.

68 Bausewein C, Jolley C, Reilly C, et al. Development, effectiveness and costeffectiveness of a new out-patient Breathlessness Support Service: study protocol of a phase III fast-track randomised controlled trial. BMC Pulm Med 2012;12:58.

69 Kachuik L, Amjadi K. An inter-professional dyspnea management clinic: collaborating to manage shortness of breath. Journal of Thoracic Oncology 2011;6(SUPPL. 2):S579-S80.

70 McMahon D, McAleer C, O'Leary N. Retrospective study of referrals to a specialist palliative care breathlessness management programme our lady's hospice and care services (OLH\&CS) dublin and of patient outcomes. Palliative Medicine 2016;30:NP253.

71 Schunk M, Berger U, Le L, et al. A mixed methods study for the evaluation of a breathlessness support service in Germany: Research protocol. Palliative Medicine 2016;30:NP258.

72 Scullion JE, Henry C. A multidisciplinary approach to managing breathlessness in lung cancer. International Journal of Palliative Nursing 1998;4:65-9.

73 Ung YC, Evans W, Gatto A, et al. Implementing dyspnea management: a quality improvement project for patients with lung cancer in Ontario, Canada. Journal of Thoracic Oncology 2013;8:\$1010 